\title{
Long-term safety, tolerability, and efficacy of evolocumab in patients with heterozygous familial hypercholesterolemia
}

\author{
G. Kees Hovingh, MD, PhD, MBA* , Frederick J. Raal, MBBCh, PhD, Ricardo Dent, MD ${ }^{1}$, \\ Claudia Stefanutti, MD, PhD, Olivier Descamps, MD, PhD, Luis Masana, MD, PhD, \\ Armando Lira, MD, Ian Bridges, MSc, Blai Coll, MD, David Sullivan, MD
}

\begin{abstract}
Department of Vascular Medicine, Academisch Medisch Centrum, Amsterdam, The Netherlands (Dr Hovingh); Faculty of Health Sciences, Department of Medicine, University of the Witwatersrand, Johannesburg, South Africa (Dr Raal); Clinical Development Department, Amgen (Europe) GmbH, Zug, Switzerland (Dr Dent); Department of Molecular Medicine, Umberto I Hospital, Sapienza University of Rome, Rome, Italy (Dr Stefanutti); Centres Hospitaliers Jolimont, Lipid Clinic, Haine-Saint-Paul, Belgium (Dr Descamps); Internal Medicine Department, Hospital Universitari Sant Joan, Lipids and Arteriosclerosis Research Unit, Universitat Rovira i Virgili, IISPV, CIBERDEM, Reus, Spain (Dr Masana); Clinical Development Department, Amgen Inc, One Amgen Center Drive, Thousand Oaks, CA, USA (Drs Lira and Coll); Biostatistics Department, Amgen Ltd, Cambridge, United Kingdom (Mr Bridges); and Department of Clinical Biochemistry, Royal Prince Alfred Hospital, Camperdown, New South Wales, Australia (Dr Sullivan)
\end{abstract}

KEYWORDS:

PCSK9;

LDL-C;

Familial

hypercholesterolemia;

Safety;

Tolerability;

Monoclonal antibody
BACKGROUND: Evolocumab, a fully human monoclonal antibody against proprotein convertase subtilisin/kexin type 9, is safe and effective when dosed biweekly (Q2W) or monthly (QM) in patients with heterozygous familial hypercholesterolemia $(\mathrm{HeFH})$ as demonstrated in two 12-week trials: Reduction of LDL-C With PCSK9 Inhibition in Heterozygous Familial Hypercholesterolemia Disorder (RUTHERFORD; phase 2) and RUTHERFORD-2 (phase 3).

OBJECTIVE: The objective of the study was to evaluate long-term efficacy, safety, and tolerability of evolocumab during open-label extension trials.

METHODS: Patients completing parent trials were re-randomized 2:1 to evolocumab plus standard of care (SOC) or SOC alone for 52 weeks (Open-Label Study of Long-term Evaluation Against LDL-C [OSLER-1]) or 48 weeks (OSLER-2). Evolocumab dosing was $420 \mathrm{mg}$ QM (OSLER-1) and $140 \mathrm{mg}$ Q2W or $420 \mathrm{mg}$ QM (OSLER-2). A pooled analysis of OSLER data was performed from this subset of HeFH patients.

RESULTS: Four hundred forty HeFH patients from RUTHERFORD $(\mathrm{n}=147)$ and RUTHERFORD-2 ( $\mathrm{n}=293)$ (mean [standard deviation] age 51 [12] years, 58\% male, 90\% White) were randomized to evolocumab plus SOC $(n=289)$ or SOC $(n=151)$. The 48-week period was completed by 425 patients (96.6\%). Eight patients discontinued evolocumab plus SOC (2.8\%) and 7

\footnotetext{
This work was supported by Amgen Inc.

${ }^{1}$ Present address: Esperion Therapeutics, 3891 Ranchero Drive, Suite 150, Ann Arbor, MI 48108, USA.

* Corresponding author. Department of Vascular Medicine, Academisch Medisch Centrum, Meibergdreef 9, Amsterdam 1105 AZ, The Netherlands.
}

\footnotetext{
E-mail address: g.k.hovingh@amc.uva.nl

Submitted March 31, 2017. Accepted for publication September 16, 2017.
} 
discontinued SOC (4.6\%). Compared to parent study baseline, patients receiving evolocumab plus SOC experienced a mean $53.6 \%$ reduction in low-density lipoprotein cholesterol after 48 weeks. No patient experienced an adverse event leading to permanent evolocumab discontinuation during the 1-year SOC-controlled period. Serious adverse event rates were similar between groups (evolocumab plus SOC, $7.3 \%$; SOC, $8.6 \%$ ).

CONCLUSION: Continued use of evolocumab added to SOC in patients with HeFH yields persistent and marked low-density lipoprotein cholesterol reductions during 48 weeks of follow-up. Long-term dosing of evolocumab with SOC was safe and well tolerated.

(C) 2017 National Lipid Association. All rights reserved.

\section{Introduction}

Heterozygous familial hypercholesterolemia $(\mathrm{HeFH})$ is a common inherited disorder, characterized by elevated plasma low-density lipoprotein cholesterol (LDL-C) concentrations. A pathogenic mutation in the LDL receptor gene (LDLR) is identified in more than $90 \%$ of the genetically confirmed HeFH cases, ${ }^{1}$ followed by mutations in the apolipoprotein B gene (APOB; 5\%) and the proprotein convertase subtilisin/ kexin type 9 gene (PCSK9; 1\%), as well as rare mutations in other genes such as the apolipoprotein $\mathrm{E}$ gene and the signal transducing adaptor family member 1 gene. $^{2-5}$

The prevalence of $\mathrm{HeFH}$ varies in different populations; however, large studies conducted in the United States and different countries in Europe have shown that approximately 1 in 200 to 300 people has HeFH. This prevalence translates to a worldwide estimated total of approximately 34 million HeFH patients. ${ }^{6,7}$

Patients with $\mathrm{HeFH}$ are at increased risk for premature cardiovascular disease, and lipid-lowering therapies are therefore the cornerstone of treatment for HeFH. However, most $\mathrm{HeFH}$ patients are either not treated at all or do not reach their guideline recommended LDL-C target levels even when treated with intensive statin therapy and ezetimibe. ${ }^{7-11}$ Statin treatment results in an almost $50 \%$ reduction of cardiovascular disease morbidity and mortality in this population ${ }^{8-12}$; however, even among statin-treated patients, a considerable residual cardiovascular disease risk remains, which underlines the need for additional therapies to lower LDL-C levels. ${ }^{13-16}$

Evolocumab, a monoclonal antibody against PCSK9, is a relatively novel lipid-lowering agent. In 12-week intervention studies, evolocumab has been shown to safely lower LDL-C levels by $56 \%$ to $61 \%$ compared with placebo when dosed $140 \mathrm{mg}$ biweekly (Q2W) or $420 \mathrm{mg}$ monthly (QM). This LDL-C lowering was observed in different patient categories, including $\mathrm{HeFH}$ patients who were receiving background lipid-lowering therapy, as demonstrated in 2 randomized, double-blind, placebo-controlled trials (ie, Reduction of LDL-C With PCSK9 Inhibition in Heterozygous Familial Hypercholesterolemia Disorder [RUTHERFORD], a phase 2 study, and RUTHERFORD2, a phase 3 study). ${ }^{17,18}$

To evaluate the long-term effects of evolocumab, patients completing the RUTHERFORD or
RUTHERFORD-2 studies were eligible to enter the evolocumab open-label extension (OLE) trial program, which comprised 2 trials: Open-Label Study of Longterm Evaluation Against LDL-C (OSLER)-1, in which patients completing evolocumab phase 2 trials were enrolled, and OSLER-2, in which patients completing evolocumab phase 3 trials were enrolled.

We evaluated the long-term efficacy, safety, and tolerability of evolocumab during the 1-year SOC-controlled period of OSLER-1 and OSLER-2 in patients with HeFH from RUTHERFORD and RUTHERFORD-2.

\section{Methods}

A pooled analysis was performed using the data obtained from patients who entered the OSLER program after completing RUTHERFORD or RUTHERFORD2. ${ }^{17,18}$ Patients were diagnosed with $\mathrm{HeFH}$ based on Simon Broome criteria, ${ }^{19}$ and/or with a genetic confirmation (defined as the presence of a pathogenic mutation in either LDLR, APOB, or PCSK9). Among patients entering the OSLER program, a total of 351 of 440 patients $(79.8 \%)$ were diagnosed with definite $\mathrm{HeFH}$ $(n=234$ [81.0\%], evolocumab plus SOC and $n=117$ [77.5\%], SOC alone), based on Simon Broome criteria. Possible $\mathrm{HeFH}$ was diagnosed in the remaining 55 and 34 patients, respectively. Genetic confirmation was ultimately obtained in 304 of the total of 368 patients (83\%) who underwent genetic testing in RUTHERFORD, RUTHERFORD-2, or the OSLER program. Genetic testing was performed by Progenika Inc (Medford, MA); samples were sequenced for mutations in the whole LDLR gene, including large deletions or rearrangements, exon 26 of the APOB gene, and for all 12 exons and promoter region of the PCSK9 gene. Ten of these patients were found to have compound $\mathrm{HeFH}$, characterized by a more severe form of hypercholesterolemia and clinical sequelae. ${ }^{20-22}$ Inclusion criteria for entry into the parent study included an LDL-C serum concentration of $\geq 2.6 \mathrm{mmol} / \mathrm{L}(100 \mathrm{mg} / \mathrm{dL})$ at baseline despite statin therapy with or without ezetimibe. In RUTHERFORD, patients received evolocumab doses of 350 or $420 \mathrm{mg}$ QM. ${ }^{17}$ In RUTHERFORD-2, patients received evolocumab doses of $140 \mathrm{mg}$ Q2W or $420 \mathrm{mg}$ QM. ${ }^{18}$ 
Lipid and apolipoprotein measurements conducted during each parent trial and during the OSLER program were analyzed at a central laboratory that met applicable standards according to the Centers for Disease Control and the National Heart, Lung, and Blood Institute. ${ }^{23}$ In RUTHERFORD, LDL-C levels were measured using preparative ultracentrifugation and calculated with the Friedewald formula. ${ }^{17}$ In RUTHERFORD-2, LDL-C levels were calculated using the Friedewald formula; if the calculated LDL-C was $\leq 1.0 \mathrm{mmol} / \mathrm{L}(40 \mathrm{mg} / \mathrm{dL})$, or triglyceride levels were $\geq 4.5 \mathrm{mmol} / \mathrm{L}$ ( $400 \mathrm{mg} / \mathrm{dL})$, additional testing via preparative ultracentrifugation was performed. ${ }^{18}$ For the current analysis of data from the OSLER program, LDL-C levels were calculated using the Friedewald formula.

In OSLER, patients were rerandomized 2:1 to receive evolocumab plus standard of care (SOC) or SOC alone for 52 weeks in OSLER-1 or 48 weeks in OSLER-2. (After the 1-year SOC-controlled period currently reported, all patients continuing in OSLER-1 or OLSER-2 received evolocumab therapy.) After enrollment, patients randomized to evolocumab plus SOC were followed in the clinic, whereas patients randomized to SOC alone were followed with a combination of clinic visits and phone calls. In OSLER-1 and OLSER-2, patients receiving evolocumab plus SOC were scheduled to visit the clinic 13 and 8 times, respectively, whereas patients receiving SOC alone were scheduled to visit the clinic 6 and 4 times, respectively. The present analysis includes data from $\mathrm{HeFH}$ patients who completed up to 48 weeks of OSLER-1 and OSLER-2. The median (min, max) durations of study exposure in the evolocumab plus SOC and SOC alone arms were 48.2 $(14.4,57.1)$ and $48.5(30.9,57.1)$ weeks, respectively. Evolocumab dosing was $420 \mathrm{mg}$ QM in OSLER-1 and $140 \mathrm{mg}$ Q2W or $420 \mathrm{mg} \mathrm{QM}$ in OSLER-2. Because the dosing regimens are considered clinically equivalent ${ }^{18}$ and to accommodate patient preference, patients were allowed to select which dosing regimen they wanted to administer (Q2W or QM). Written informed consent was provided by each patient, and the individual study protocols were approved by each respective institutional review board.

\section{Efficacy and safety endpoints}

In this pooled analysis, mean percent changes in lipid parameters from parent study baseline to week 48 of the OLE trials were evaluated as the primary efficacy outcome. Overall adverse event (AE) rates, serious AE rates, rates of discontinuation for AE, laboratory assessments, and AEs of interest associated with lipid-lowering therapies (diabetes, muscle events, and neurocognitive events) were analyzed as safety outcomes. Potential diabetes-related events were identified using the hyperglycemia/new-onset diabetes mellitus narrow search Standard Medical Dictionary of Regulatory Activities (MedDRA) Query. Potential musclerelated events were identified using the rhabdomyolysismyopathy broad search Standard MedDRA Query. Neurocognitive events were identified using the following high-level group terms: deliria (including confusion), cognitive and attention disorders and disturbances, dementia and amnestic conditions, disturbances in thinking and perception, and mental impairment disorders. Safety data were collected through the end of the 1-year SOCcontrolled treatment period for both OSLER trials. AEs were coded according to the MedDRA version current at the time of database lock for RUTHERFORD or RUTHERFORD-2. The immunogenicity of evolocumab was evaluated in all patients by means of an electrochemiluminescent bridging immunoassay for the detection of binding anti-drug antibodies. For patients whose sera tested positive in the immunoassay, an in vitro biological assay was performed to detect neutralizing antibodies.

\section{Statistical analysis}

All analyses conducted were descriptive in nature and included all subjects who completed RUTHERFORD or RUTHERFORD-2 and were randomized in the OLE studies. No imputation was performed for missing data. Continuous data were summarized at the baseline measurement in the parent studies using mean and standard deviation (SD; or median and quartiles) and was summarized during the OLE study using mean and standard error. Categorical data were summarized using the absolute number and percentage. Mean percent change from parent study baseline to week 48 of the OLE study was summarized by randomized treatment group.

\section{Results}

A total of 440 patients from RUTHERFORD and RUTHERFORD-2 entered the OSLER program (Fig. 1). These patients included 147 of 168 patients $(87.5 \%)$ randomized in RUTHERFORD and 293 of 331 patients

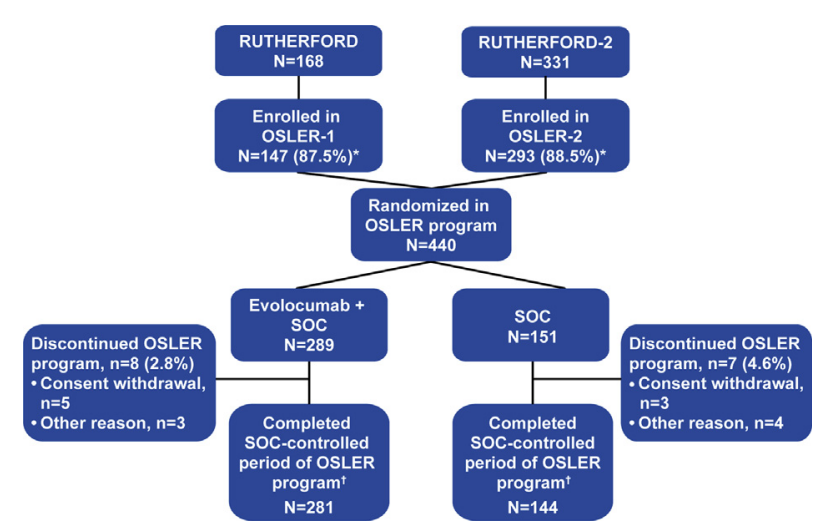

Figure 1 Patient disposition in the parent RUTHERFORD trials and the open-label extension OSLER trials. * Of the 59 patients who did not enter the OSLER program, $40(67 \%)$ cited personal reasons unrelated to the study, or the level of commitment required as the reason for not entering. ${ }^{\dagger}$ Cut-off dates for the 1year SOC-controlled period were October 2014 (OSLER-1) and April 2015 (OSLER-2). SOC, standard of care. 
(88.5\%) randomized in RUTHERFORD-2. In the OSLER program, 289 patients were randomized to receive evolocumab plus SOC and 151 patients were randomized to SOC alone. As of October 2014 (OSLER-1) and April 2015 (OSLER-2), 425 patients (96.6\%) completed the 1-year SOC-controlled period. Fifteen patients (3.4\%) discontinued the OLE because of consent withdrawal $(n=8)$ or other reasons $(n=7)$. In the evolocumab plus SOC arm, other reasons were patient desire to discontinue participation $(\mathrm{n}=1)$, moving out of country $(\mathrm{n}=1)$, and desire to stop receiving injections $(\mathrm{n}=1)$. In the SOC arm, other reasons were patient desire to discontinue participation in all patients $(n=4)$. No deaths were recorded among patients not completing the study. Eight patients who discontinued $(2.8 \%)$ were receiving evolocumab plus SOC and 7 patients $(4.6 \%)$ were receiving SOC alone.

Baseline characteristics are summarized in Table 1. In the pooled population, $57.5 \%$ of patients were men,
$90.2 \%$ were White, and the mean (SD) age of participants was 50.8 (12.4) years. Coronary artery disease was present in $28.0 \%$ of patients and cerebrovascular or peripheral arterial disease was present in $13.9 \%$ of patients. Mean (SD) baseline LDL-C was 4.0 (1.2) mmol/ L, with $78.9 \%$ of patients receiving high-intensity statin therapy (atorvastatin $40-80 \mathrm{mg}$ or rosuvastatin 20 $40 \mathrm{mg}$ ) and $64.3 \%$ of patients receiving ezetimibe in addition to statin therapy.

\section{Efficacy}

Compared with parent study baseline, patients receiving evolocumab plus SOC during the OLE experienced a mean (standard error [SE]) 53.6 (1.6)\% reduction in calculated LDL-C after 48 weeks, compared with a mean (SE) increase of $2.1(2.1) \%$ in the SOC alone arm (Fig. 2).

Table 1 Baseline characteristics*

\begin{tabular}{|c|c|c|c|}
\hline Characteristic & $\begin{array}{l}\text { Evolocumab }+\mathrm{SOC} \\
(\mathrm{N}=289)\end{array}$ & SOC $(N=151)$ & Total $(\mathrm{N}=440)$ \\
\hline Mean age (SD), y & $50.2(12.4)$ & $52.0(12.2)$ & $50.8(12.4)$ \\
\hline \multicolumn{4}{|l|}{ Sex, $n(\%)$} \\
\hline Male & $165(57.1)$ & $88(58.3)$ & $253(57.5)$ \\
\hline Female & $124(42.9)$ & $63(41.7)$ & $187(42.5)$ \\
\hline \multicolumn{4}{|l|}{ Race or ethnicity, n (\%) } \\
\hline White & $261(90.3)$ & $136(90.1)$ & $397(90.2)$ \\
\hline Black & $6(2.1)$ & $1(0.7)$ & $7(1.6)$ \\
\hline Asian & $16(5.5)$ & $8(5.3)$ & $24(5.5)$ \\
\hline Hispanic & $3(1.0)$ & $3(2.0)$ & $6(1.4)$ \\
\hline Cardiovascular disease, n (\%) & $99(34.3)$ & $51(33.8)$ & $150(34.1)$ \\
\hline Coronary artery disease & $79(27.3)$ & $44(29.1)$ & $123(28.0)$ \\
\hline Cerebrovascular or peripheral arterial disease & $38(13.1)$ & $23(15.2)$ & $61(13.9)$ \\
\hline \multicolumn{4}{|l|}{ Lipid parameters $^{\dagger}$} \\
\hline LDL-C, mmol/L, calculated & $4.0(1.2)$ & $3.9(1.0)$ & $4.0(1.2)$ \\
\hline $\mathrm{Lp}(\mathrm{a}), \mathrm{nmol} / \mathrm{L}$, median $(01,03)$ & $63.0(23.0,196.0)^{\ddagger}$ & $44.0(17.0,194.0)$ & $58.0(21.0,195.0)$ \\
\hline ApoB, g/L & $1.2(0.3)^{\ddagger}$ & $1.2(0.3)$ & $1.2(0.3)$ \\
\hline $\mathrm{HDL}-\mathrm{C}, \mathrm{mmol} / \mathrm{L}$ & $1.3(0.4)$ & $1.3(0.4)$ & $1.3(0.4)$ \\
\hline Non-HDL-C, mmol/L & $4.7(1.4)$ & $4.5(1.1)$ & $4.6(1.3)$ \\
\hline $\mathrm{TG}, \mathrm{mmol} / \mathrm{L}$ & $1.4(0.7)$ & $1.4(0.8)$ & $1.4(0.8)$ \\
\hline PCSK9, nmol/L & $7.0(2.4)$ & $6.9(2.4)$ & $7.0(2.4)$ \\
\hline \multicolumn{4}{|l|}{ Statin treatment intensity, ${ }^{\S} \mathrm{n}(\%)$} \\
\hline High & $234(81.0)$ & $113(74.8)$ & $347(78.9)$ \\
\hline Moderate & $50(17.3)$ & $31(20.5)$ & $81(18.4)$ \\
\hline Low & $4(1.4)$ & $7(4.6)$ & $11(2.5)$ \\
\hline Unknown & $1(0.3)$ & 0 & $1(0.2)$ \\
\hline Ezetimibe, n (\%) & $187(64.7)$ & $96(63.6)$ & $283(64.3)$ \\
\hline
\end{tabular}

ApoB, apolipoprotein B; HDL-C, high-density lipoprotein cholesterol; LDL-C, low-density lipoprotein cholesterol; Lp(a), lipoprotein(a); PCSK9, proprotein convertase subtilisin/kexin type 9; SOC, standard of care; TG, triglycerides.

${ }^{*}$ At the start of the parent study for the pooled population evaluated in the current analysis.

$\dagger$ Mean (standard deviation) unless otherwise noted.

$\ddagger n=286$.

$\S$ Per American College of Cardiology/American Heart Association guidelines ${ }^{24}$ : high intensity, atorvastatin $40-80 \mathrm{mg}$, rosuvastatin $20-40 \mathrm{mg}$; moderate intensity, atorvastatin $10-20 \mathrm{mg}$, rosuvastatin $5-10 \mathrm{mg}$, simvastatin $20-40 \mathrm{mg}$, pravastatin $40-80 \mathrm{mg}$, lovastatin $40 \mathrm{mg}$, fluvastatin XL $80 \mathrm{mg}$, fluvastatin $40 \mathrm{mg}$ twice daily, pitavastatin $2-4 \mathrm{mg}$; low intensity, simvastatin $10 \mathrm{mg}$, pravastatin $10-20 \mathrm{mg}$, lovastatin $20 \mathrm{mg}$, fluvastatin 20-40 mg, pitavastatin $1 \mathrm{mg}$. 


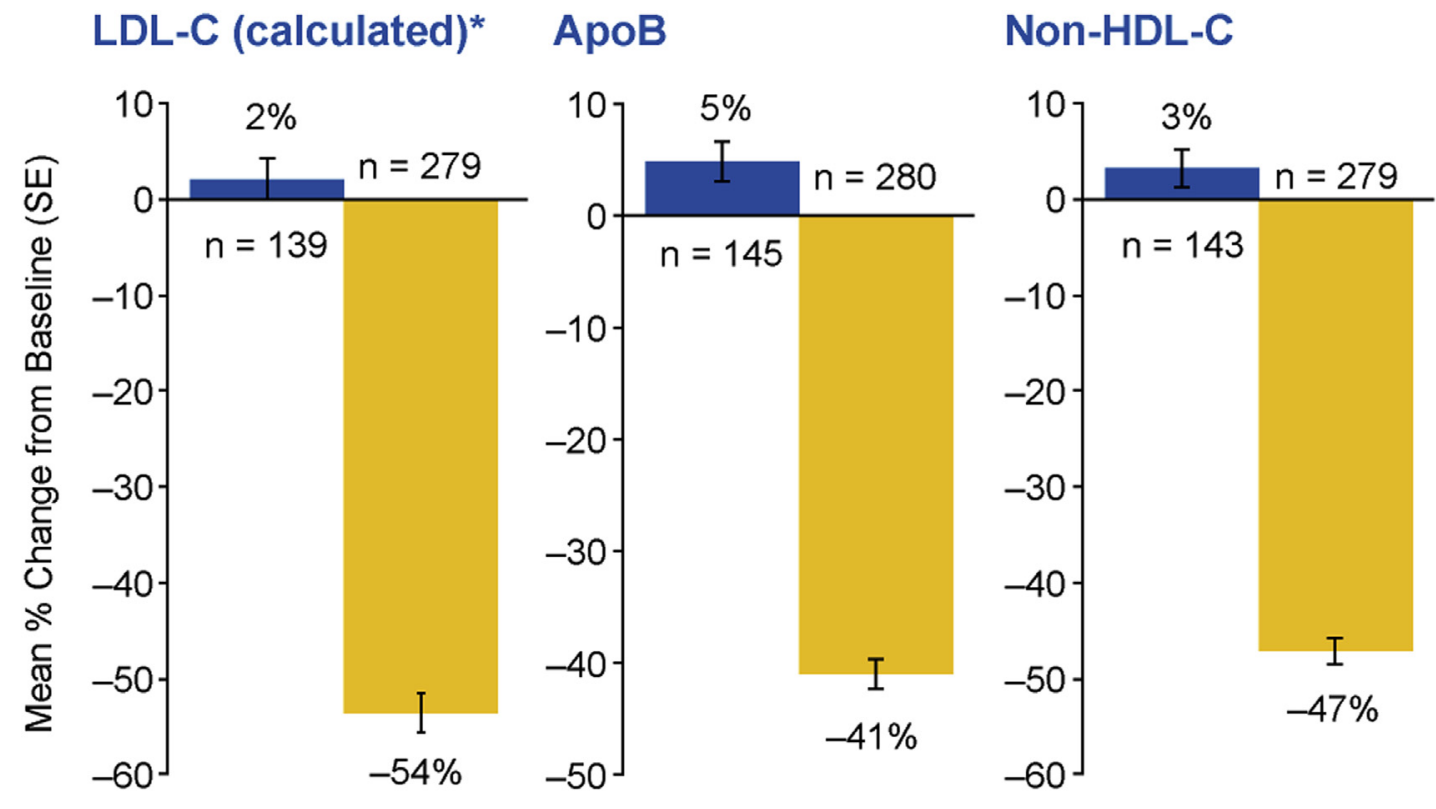

\section{Triglycerides}

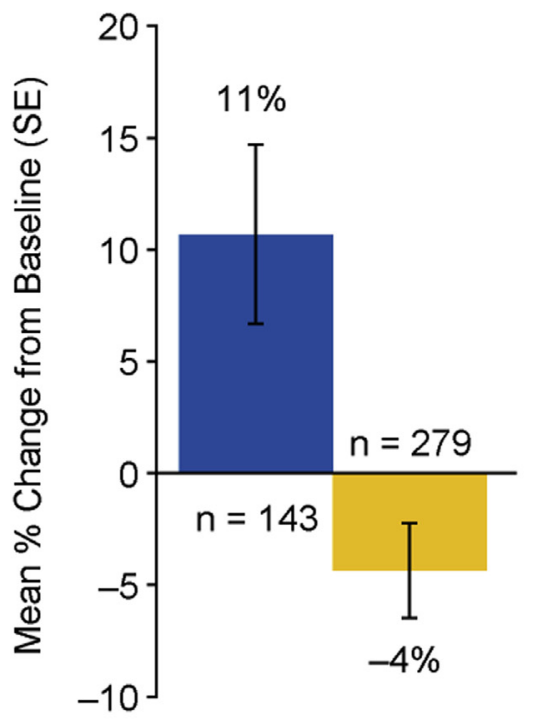

HDL-C

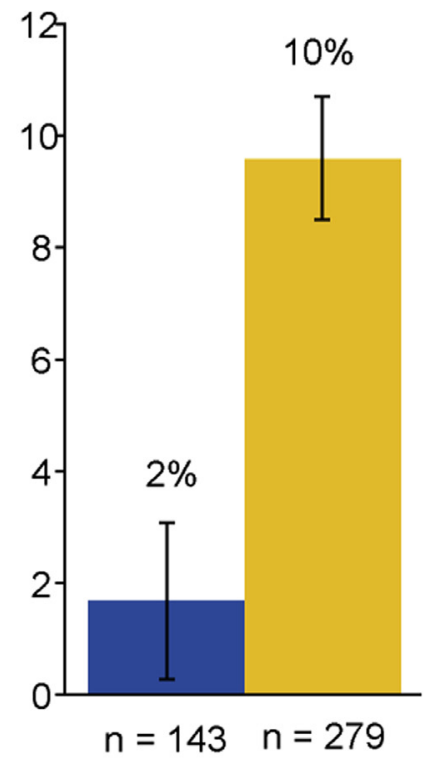

$\operatorname{Lp}(a)$

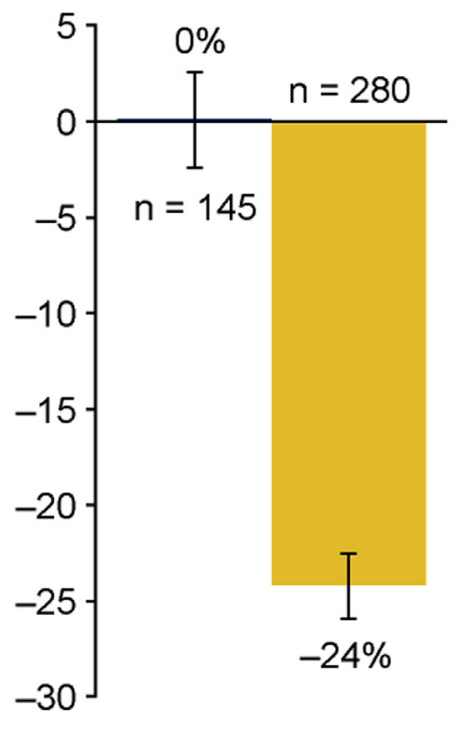

SOC alone Evolocumab $+\mathrm{SOC}$

Figure 2 Mean percent change in lipid parameters from parent study baseline to week 48 of the OLE trials. *Mean (standard error) changes from baseline in LDL-C levels were $-2.1(0.07) \mathrm{mmol} / \mathrm{L}$ in the evolocumab plus SOC arm and $0.03(0.09) \mathrm{mmol} / \mathrm{L}$ in the SOC alone arm. ApoB, apolipoprotein B; HDL-C, high-density lipoprotein cholesterol; LDL-C, low-density lipoprotein cholesterol; Lp(a), lipoprotein(a); SOC, standard of care.

Mean (SE) 48-week LDL-C levels were $1.9(0.07)$ $\mathrm{mmol} / \mathrm{L}$ in the evolocumab plus SOC arm and $3.9(0.11)$ $\mathrm{mmol} / \mathrm{L}$ in the SOC alone arm. Mean (SE) changes from baseline in LDL-C levels were $-2.1(0.07) \mathrm{mmol} / \mathrm{L}$ in the evolocumab plus SOC arm and $0.03(0.09) \mathrm{mmol} / \mathrm{L}$ in the SOC alone arm. Reductions in ApoB, non-high-density lipoprotein cholesterol (non-HDL-C), triglycerides, and lipoprotein(a) were also observed in the evolocumab plus SOC arm, along with an increase in HDL-C (Fig. 2).

The pattern of LDL-C serum levels over time are presented in Figure 3. Among patients who had received evolocumab during the parent trials, continuing on evolocumab plus SOC in the OLE trials led to sustained reductions in LDL-C. Among patients who received control treatment 


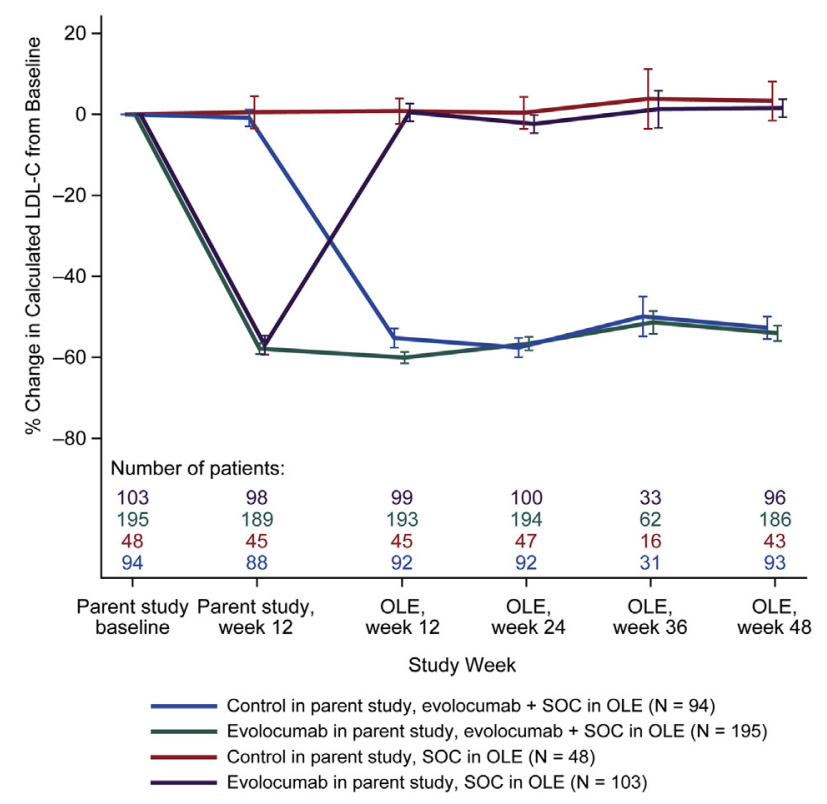

Figure 3 Mean percent change from baseline in calculated LDL-C by scheduled visit and treatment group. Data at each time point represent all evolocumab doses combined $(140 \mathrm{mg}$ every 2 weeks [Q2W], $350 \mathrm{mg}$ monthly [QM], and $420 \mathrm{mg}$ QM at parent study baseline and week 12; and $140 \mathrm{mg}$ Q2W and $420 \mathrm{mg} \mathrm{QM}$ at OLE weeks 12-48). At week 36, lipid parameters were only assessed in OSLER-1. Vertical lines represent the standard error around the mean. Plot is based on observed data and no imputation is used for missing values. LDL-C, low-density lipoprotein cholesterol; OLE, open-label extension; SOC, standard of care.

during the parent trials, the addition of evolocumab to SOC in the OLE trials led to reductions in LDL-C that were consistent with sustained reductions in patients who had received evolocumab during the parent trials. In patients who received evolocumab in the parent trials and SOC in the OLE, LDL-C returned to, and did not exceed, baseline levels during the OLE.

\section{Safety}

No patient experienced an $\mathrm{AE}$ leading to permanent discontinuation of evolocumab treatment during the 1-year SOC-controlled period of the OLE trials. Overall AE rates reported for evolocumab plus SOC and SOC alone were $79.9 \%$ of patients and $66.9 \%$ of patients, respectively (Table 2).

AEs, categorized by system-organ-class that occurred with a $\geq 5 \%$ absolute frequency in the evolocumab plus SOC arm compared with the SOC alone arm and reported here as the percentage of patients who experienced 1 or more of these events, were infections and infestations (47.8\% vs $37.1 \%)$; musculoskeletal and connective tissue disorders (33.2\% vs $21.9 \%$ ); general disorders and administration site conditions ( $25.3 \%$ vs $7.3 \%)$; gastrointestinal disorders (19.7\% vs $12.6 \%$ ); and nervous system disorders (14.5\% vs $7.9 \%$ ). Individual AEs occurring in $\geq 5 \%$ of patients in either arm are detailed in Table 2. Serious AEs were well balanced in both treatment arms $(7.3 \%$, evolocumab plus SOC and $8.6 \%$, SOC alone).

Hyperglycemia/new-onset diabetes mellitus events and neurocognitive events were also balanced between arms (Table 2). The incidence of muscle-related events was more frequently observed in patients randomized to evolocumab plus SOC, compared with SOC alone (10.0\% vs $4.6 \%)$. All events were of grade 1 or 2 severity, except one grade 3 event of myalgia, which began as a grade 1 event starting 2 days after evolocumab dosing was initiated and worsened to a grade 3 event over the subsequent 4 months; this event was ongoing at the final $\mathrm{AE}$ evaluation. Evolocumab was withheld for 1 dose based on this change in AE severity and then resumed. Of the 29 patients who experienced a potential muscle-related $\mathrm{AE}$ while receiving evolocumab, 9 patients $(31 \%)$ experienced a creatine kinase (CK) elevation of $>1$ times the upper limit of normal $(X \mathrm{ULN})$ that was temporally associated with the muscle-related event, including 1 patient who experienced a CK elevation of $>10 \times$ ULN at 1 visit. This patient continued evolocumab after the event and the CK level returned to normal. No CK elevations of $>5 \times$ ULN occurred in patients receiving SOC alone. Most muscle-related events had resolved while patients remained on study. At the time of data cut-off, of 37 events occurring in 29 evolocumab plus SOC-treated patients, 20 events (54\%) had resolved; of 7 events occurring in 7 SOC-treated patients, 5 events $(71 \%)$ had resolved.

Liver enzyme elevations of $>3 \times$ ULN occurred in 9 patients receiving evolocumab plus SOC (3.1\%) and in no patients receiving SOC alone (Table 2). Total bilirubin elevations of $>2 \times$ ULN occurred in 2 patients receiving evolocumab plus SOC $(0.7 \%)$ and no patients receiving SOC alone. All elevations resolved by the end of the OLE while the patients continued receiving evolocumab; no patient had evolocumab dosing withheld temporarily or permanently because of these elevations. No patient had concomitant liver enzyme and bilirubin elevation.

During the OLE trials, no neutralizing antibodies were detected. Binding antibodies were detected at 1 visit (OLE week 4$)$ in 1 patient who was receiving SOC alone $(0.7 \%)$. This patient had received evolocumab during the parent study and the binding antibodies were detected 8 weeks after the last evolocumab dose. No AEs were recorded for this patient at the time of binding antibody detection.

\section{Discussion}

Long-term dosing of evolocumab in addition to SOC provided sustained LDL-C lowering consistent with reductions observed during the randomized controlled phase 2 and 3 clinical trials and was well tolerated.

Reductions in lipid levels were stable over 48 weeks and discontinuation of evolocumab resulted in a return to baseline levels. The mean LDL-C reduction from baseline demonstrated at this 48 -week time point $(53.6 \%)$ is lower 
Table 2 Adverse events and laboratory investigations

\begin{tabular}{|c|c|c|}
\hline Adverse events, n (\%) & Evolocumab + SOC $(\mathrm{N}=289)$ & SOC $(N=151)$ \\
\hline Any & $231(79.9)$ & $101(66.9)$ \\
\hline Serious & $21(7.3)$ & $13(8.6)$ \\
\hline Leading to study drug discontinuation & 0 & NA \\
\hline \multicolumn{3}{|l|}{ Adverse events of interest } \\
\hline Hyperglycemia/new-onset diabetes mellitus events* & $3(1.0)$ & $3(2.0)$ \\
\hline Muscle events ${ }^{\dagger}$ & $29(10.0)$ & $7(4.6)$ \\
\hline Myalgia & $16(5.5)$ & $6(4.0)$ \\
\hline Muscular weakness & $1(0.3)$ & 0 \\
\hline Muscle rupture & $2(0.7)$ & 0 \\
\hline Musculoskeletal pain & $9(3.1)$ & $1(0.7)$ \\
\hline Renal impairment & $1(0.3)$ & 0 \\
\hline Blood CK increased & $1(0.3)$ & 0 \\
\hline Neurocognitive events ${ }^{\ddagger}$ & $1(0.3)$ & 0 \\
\hline \multicolumn{3}{|c|}{ Adverse events occurring in $\geq 5 \%$ of patients in either treatment arm } \\
\hline Nasopharyngitis & $49(17.0)$ & $9(6.0)$ \\
\hline Influenza & $30(10.4)$ & $10(6.6)$ \\
\hline Arthralgia & $26(9.0)$ & $6(4.0)$ \\
\hline Upper respiratory tract infection & $25(8.7)$ & $15(9.9)$ \\
\hline Back pain & $21(7.3)$ & $4(2.6)$ \\
\hline Headache & $19(6.6)$ & $6(4.0)$ \\
\hline Myalgia & $16(5.5)$ & $6(4.0)$ \\
\hline Fatigue & $16(5.5)$ & 0 \\
\hline \multicolumn{3}{|l|}{ Muscle or liver enzyme elevations at any post-baseline visit } \\
\hline $\mathrm{CK}>5 \times$ ULN and $\leq 10 \times$ ULN & 0 & 0 \\
\hline CK $>10 \times$ ULN & $1(0.3)$ & 0 \\
\hline ALT or AST $>3 \times$ ULN and $\leq 5 \times$ ULN & $8(2.8)$ & 0 \\
\hline ALT or AST $>5 \times$ ULN & $1(0.3)$ & 0 \\
\hline Total bilirubin $>2 \times$ ULN & $2(0.7)$ & 0 \\
\hline
\end{tabular}

compared with the reduction demonstrated in RUTHERFORD-2, where a $61.2 \%$ reduction was observed at weeks 10 and 12 in patients randomized to $140 \mathrm{mg} \mathrm{Q} 2 \mathrm{~W}$ evolocumab and $63.3 \%$ for 420 QM evolocumab. ${ }^{18}$ This difference arises from the averaging of week 10 and 12 data in RUTHERFORD-2, which captures the time averaged LDL-C reduction over the dosing interval for both the Q2W and QM dosing regimens; in the present analysis, efficacy was assessed only at a single time point (reflecting 4-week postdose in the evolocumab 420 QM-treated patients and 2-week postdose in the $140 \mathrm{mg}$ Q2W-treated patients). Approximately, two-thirds of patients in OSLER (-1 and -2 combined) received monthly evolocumab dosing.

The incidence of serious AEs were balanced between the evolocumab plus SOC and SOC alone arms, occurring in $7.3 \%$ of evolocumab-treated patients and $8.6 \%$ of the control group. Overall AE rates were numerically higher in the evolocumab-treated group than the control group (79.9\% and $66.9 \%$, respectively). This difference may be because of potential reporting biases associated with an open-label trial design. ${ }^{25}$ In addition, in OSLER, patients receiving SOC alone had fewer in-clinic visits than patients receiving evolocumab. Most importantly, no patients in the evolocumab-treated group permanently discontinued drug because of an AE during the 1-year SOC-controlled period.

Several AEs are of interest in patients receiving lipidlowering therapy, including diabetes, neurocognitive events, and muscle-related symptoms. Statins have been shown to increase the risk for the development of diabetes. ${ }^{26-28}$ Recently, Mendelian randomization studies have shown a positive association between PCSK9 loss of function gene variants and increased risk of diabetes. ${ }^{29}$ Although the clinical implications of this observation need further confirmation, we did not observe such an effect of evolocumab. However, rates of potential diabetesrelated AEs were low in this relatively small study $(1.0 \%$ and $2.0 \%$ in the evolocumab plus SOC and SOC alone groups, respectively). Whether evolocumab treatment 
results in the development of new-onset diabetes is 1 of the points of interest evaluated in the large-scale Further Cardiovascular Outcomes Research With PCSK9 Inhibition in Subjects with Elevated Risk trial (FOURIER). ${ }^{30}$ In FOURIER, 27,564 patients aged 40 to 85 years with clinically evident cardiovascular disease and high-risk characteristics were randomized to receive evolocumab (either $140 \mathrm{mg}$ Q2W or $420 \mathrm{mg}$ QM per patient preference) or placebo. With a median follow-up of 26 months, rates of adjudicated cases of new-onset diabetes did not differ significantly between the 2 groups.

In the 1-year SOC-controlled period of the present study, only 1 neurocognitive event was reported-occurring in a patient in the evolocumab plus SOC arm, precluding the ability to perform a clinically meaningful comparison between groups. However, the potential effect of evolocumab on neurocognitive functioning was investigated in FOURIER patients who enrolled in the Evaluating PCSK9 Binding antibody Influence oN coGnitive HeAlth in High cardiovascular Risk Subjects (EBBINGHAUS [NCT02207634]) trial, a dedicated cognition study of more than 1900 patients. ${ }^{31}$ With a median follow-up of approximately 19 months in EBBINGHAUS, the addition of evolocumab to statin therapy did not affect cognitive function over time.

Muscle symptoms are a well-described side effect of statin therapy. In the present study, potential muscle events were reported in $10.0 \%$ of patients treated with evolocumab plus SOC and $4.6 \%$ of patients receiving SOC alone. Reasons for the numerically higher incidence in the evolocumab group are uncertain. An integrated analysis of 6026 patients randomized in phase 2 and 3 clinical trials of the evolocumab clinical trial program (Program to Reduce LDL-C and Cardiovascular Outcomes Following Inhibition of PCSK9 In Different POpulations) across various patient populations and administered as monotherapy or in combination with background lipid-lowering therapy did not demonstrate a clinically meaningful increase in muscle-related AEs associated with evolocumab. In that integrated analysis, the incidence of any musculoskeletal or connective tissue disorder was $13.7 \%$ in the integrated control arm and $14.7 \%$ in the integrated evolocumab arm. ${ }^{32}$ Patients with $\mathrm{HeFH}$ are generally younger than other hypercholesterolemic populations and typically have low rates of muscle symptoms when treated with statins or ezetimibe. ${ }^{33,34}$ Conversely, these patients are also more physically active, which may contribute to mild asymptomatic elevations in $\mathrm{CK}$ and muscle symptoms. Approximately one-third of patients who had muscle events also experienced a CK elevation of $>1 \times$ ULN that was temporally associated with the muscle-related event, including 1 patient who experienced a CK elevation of $>10 \times$ ULN. These findings may indicate muscle symptoms related to physical activity. An alternative potential explanation is unknown baseline imbalances; patients with a preponderance to muscle symptoms may have been more frequently enrolled in the cohort treated with evolocumab by chance.

This study was conducted in patients with clinical and/or genetically confirmed FH. Among patients who agreed to genotyping, $83 \%$ were found to have a mutation underlying their $\mathrm{HeFH}$ diagnosis. This identification rate is high compared with a previous study conducted in clinical $\mathrm{FH}$ patients in the United Kingdom ${ }^{35}$ and a recent study of the prevalence of mutations in patients selected for high LDL-C levels. ${ }^{12}$ The yield was similar to the rate identified in a large Netherlands study ${ }^{36}$ and the differences between the studies are most likely related to the differences in the criteria used for the identification of clinical FH.

Strengths of this analysis are the 48-week duration and the high study completion rate. A total of 425 patients (85\%) of the 499 initially randomized in RUTHERFORD and RUTHERFORD-2 enrolled in the OLE studies and completed 48 weeks of the OSLER program. A potential limitation of the analysis is the open-label design for assessing long-term tolerability issues. In addition, the sample size is not sufficient to allow for subgroup analyses.

\section{Conclusions}

This pooled analysis provides clinicians with important long-term data from 1 year of evolocumab treatment in OLE trials for 440 patients with $\mathrm{HeFH}$, a homogenous population at high risk for cardiovascular disease who required additional aggressive LDL-C reduction. This study demonstrates that long-term treatment with evolocumab is safe and well tolerated, allowing for sustained long-term LDL-C reduction.

\section{Acknowledgments}

The authors thank Mahta Nili, PhD, of Amgen, and Laura Evans, PharmD, on behalf of Amgen, for preliminary drafting and editorial support; and Tim Palmer and Jonathan Liu, PhD, of Amgen, for statistical programming.

Amgen sponsored and designed the trials and was responsible for data collection and analysis. The first draft of the article was written by the G.K.H. and Amgen. All the coauthors participated in subsequent revisions of the article. The academic authors had full access to the data and vouch for their accuracy and completeness and for the analyses as presented and for the fidelity of this report to the trial protocols. The academic authors made the decision to submit the article for publication.

\section{Financial Disclosure}

G.K.H. reports consulting and/or lecture fees from Amgen Inc, Regeneron/Sanofi, and Pfizer related to PCSK9 inhibitors, and institutional research funding related to PCSK9 inhibitor clinical trials from Amgen Inc, Sanofi, 
Eli Lilly, and Pfizer. F.J.R. reports consulting fees from Amgen Inc and Sanofi related to PCSK9 inhibitors, and institutional research funding related to PCSK9 inhibitor clinical trials from Amgen Inc and Sanofi. C.S. reports clinical trial institution research funding from Regeneron/ Sanofi and Amgen Inc, and research grants from Aegerion, Ionis, Merck, Kaneka, and Fresenius Medical Care. O.D. reports institutional research funding related to clinical trials from Sanofi and Amgen Inc, research grants from Sanofi, AstraZeneca, Amgen Inc and Merck, and honoraria for conference and advisory board from Sanofi, Amgen Inc, AstraZeneca, and Merck. L.M. reports lecture and advisory fees from Amgen Inc, Sanofi, and MSD. D.S. reports research funding from Amgen Inc, Abbott, Amarin, MSD, and Sanofi, educational program funding from Abbott, Amgen Inc, MSD, and Roche, and advisory board participation for Amgen Inc, MSD, and Sanofi Australia. A.L., I.B., and B.C. are employees and stockholders of Amgen Inc. R.D. is a former employee and stockholder of Amgen Inc and is currently employed by Esperion Therapeutics.

\section{References}

1. Leigh SE, Foster AH, Whittall RA, Hubbart CS, Humphries SE. Update and analysis of the University College London low density lipoprotein receptor familial hypercholesterolemia database. Ann Hum Genet. 2008;72(Pt 4):485-498.

2. Abifadel M, Varret M, Rabes JP, et al. Mutations in PCSK9 cause autosomal dominant hypercholesterolemia. Nat Genet. 2003;34(2): 154-156.

3. Cenarro A, Etxebarria A, de Castro-Oros I, et al. The p.Leu167del mutation in APOE gene causes autosomal dominant hypercholesterolemia by down-regulation of LDL receptor expression in hepatocytes. J Clin Endocrinol Metab. 2016;101(5):2113-2121.

4. Fouchier SW, Dallinga-Thie GM, Meijers JC, et al. Mutations in STAP1 are associated with autosomal dominant hypercholesterolemia. Circ Res. 2014;115(6):552-555.

5. Soria LF, Ludwig EH, Clarke HR, Vega GL, Grundy SM, McCarthy BJ. Association between a specific apolipoprotein B mutation and familial defective apolipoprotein B-100. Proc Natl Acad Sci U S A. 1989;86(2):587-591.

6. de Ferranti SD, Rodday AM, Mendelson MM, Wong JB, Leslie LK, Sheldrick RC. Prevalence of familial hypercholesterolemia in the 1999 to 2012 United States National Health and Nutrition Examination Surveys (NHANES). Circulation. 2016;133(11):1067-1072.

7. Nordestgaard BG, Chapman MJ, Humphries SE, et al. Familial hypercholesterolaemia is underdiagnosed and undertreated in the general population: guidance for clinicians to prevent coronary heart disease: consensus statement of the European Atherosclerosis Society. Eur Heart J. 2013;34(45):3478-3490a.

8. Neil A, Cooper J, Betteridge J, et al. Reductions in all-cause, cancer, and coronary mortality in statin-treated patients with heterozygous familial hypercholesterolaemia: a prospective registry study. Eur Heart J. 2008;29(21):2625-2633.

9. Pijlman AH, Huijgen R, Verhagen SN, et al. Evaluation of cholesterol lowering treatment of patients with familial hypercholesterolemia: a large cross-sectional study in The Netherlands. Atherosclerosis. 2010;209(1):189-194.

10. Stein EA, Ose L, Retterstol K, et al. Further reduction of low-density lipoprotein cholesterol and C-reactive protein with the addition of ezetimibe to maximum-dose rosuvastatin in patients with severe hypercholesterolemia. J Clin Lipidol. 2007;1(4):280-286.
11. Versmissen J, Oosterveer DM, Yazdanpanah M, et al. Efficacy of statins in familial hypercholesterolaemia: a long term cohort study. BMJ. 2008;337:a2423.

12. Khera AV, Won HH, Peloso GM, et al. Diagnostic yield and clinical utility of sequencing familial hypercholesterolemia genes in patients with severe hypercholesterolemia. J Am Coll Cardiol. 2016;67(22): 2578-2589.

13. Benn M, Watts GF, Tybjaerg-Hansen A, Nordestgaard BG. Familial hypercholesterolemia in the Danish general population: prevalence, coronary artery disease, and cholesterol-lowering medication. $J$ Clin Endocrinol Metab. 2012;97(11):3956-3964.

14. Mundal L, Sarancic M, Ose L, et al. Mortality among patients with familial hypercholesterolemia: a registry-based study in Norway, 19922010. J Am Heart Assoc. 2014;3(6):e001236.

15. Perak AM, Ning H, de Ferranti SD, Gooding HC, Wilkins JT, Lloyd-Jones DM. Long-term risk of atherosclerotic cardiovascular disease in US adults with the familial hypercholesterolemia phenotype. Circulation. 2016;134(1):9-19.

16. Perez de Isla L, Alonso R, Mata N, et al. Coronary heart disease, peripheral arterial disease, and stroke in familial hypercholesterolaemia: insights from the SAFEHEART registry (Spanish Familial Hypercholesterolaemia Cohort Study). Arterioscler Thromb Vasc Biol. 2016;36(9):2004-2010.

17. Raal F, Scott R, Somaratne R, et al. Low-density lipoprotein cholesterol-lowering effects of AMG 145, a monoclonal antibody to proprotein convertase subtilisin/kexin type 9 serine protease in patients with heterozygous familial hypercholesterolemia: the reduction of LDL-C with PCSK9 inhibition in heterozygous familial hypercholesterolemia disorder (RUTHERFORD) randomized trial. Circulation. 2012;126(20):2408-2417.

18. Raal FJ, Stein EA, Dufour R, et al. PCSK9 inhibition with evolocumab (AMG 145) in heterozygous familial hypercholesterolaemia (RUTHERFORD-2): a randomised, double-blind, placebo-controlled trial. Lancet. 2015;385(9965):331-340.

19. Risk of fatal coronary heart disease in familial hypercholesterolaemia. Scientific Steering Committee on behalf of the Simon Broome Register Group. BMJ. 1991;303(6807):893-896.

20. Sjouke B, Kusters DM, Kindt I, et al. Homozygous autosomal dominant hypercholesterolaemia in the Netherlands: prevalence, genotype-phenotype relationship, and clinical outcome. Eur Heart J. 2015;36:560-565.

21. Tai ES, Koay ES, Chan E, et al. Compound heterozygous familial hypercholesterolemia and familial defective apolipoprotein B-100 produce exaggerated hypercholesterolemia. Clin Chem. 2001;47(3):438-443.

22. Usifo E, Leigh SE, Whittall RA, et al. Low-density lipoprotein receptor gene familial hypercholesterolemia variant database: update and pathological assessment. Ann Hum Genet. 2012;76(5):387-401.

23. Myers GL, Cooper GR, Winn CL, Smith SJ. The Centers for Disease Control-National Heart, Lung and Blood Institute Lipid Standardization Program. An approach to accurate and precise lipid measurements. Clin Lab Med. 1989;9(1):105-135.

24. Stone NJ, Robinson JG, Lichtenstein AH, et al. 2013 ACC/AHA guideline on the treatment of blood cholesterol to reduce atherosclerotic cardiovascular risk in adults: a report of the American College of Cardiology/American Heart Association Task Force on Practice Guidelines. J Am Coll Cardiol. 2014;63(25 Pt B):2889-2934.

25. Beyer-Westendorf J, Buller H. External and internal validity of open label or double-blind trials in oral anticoagulation: better, worse or just different? Journal of thrombosis and haemostasis. J Thromb Haemost. 2011;9(11):2153-2158.

26. Rojas-Fernandez CH, Goldstein LB, Levey AI, Taylor BA, Bittner V, The National Lipid Association's Safety Task Force. An assessment by the Statin Cognitive Safety Task Force: 2014 update. J Clin Lipidol. 2014;8(3 Supp1):S5-S16.

27. Sattar N, Preiss D, Murray HM, et al. Statins and risk of incident diabetes: a collaborative meta-analysis of randomised statin trials. Lancet. 2010;375(9716):735-742.

28. Strom BL, Schinnar R, Karlawish J, Hennessy S, Teal V, Bilker WB Statin therapy and risk of acute memory impairment. JAMA Intern Med. 2015;175(8):1399-1405. 
29. Ference BA, Robinson JG, Brook RD, et al. Variation in PCSK9 and HMGCR and risk of cardiovascular disease and diabetes. $N$ Engl $J$ Med. 2016;375(22):2144-2153.

30. Sabatine MS, Giugliano RP, Keech AC, et al. Evolocumab and clinical outcomes in patients with cardiovascular disease. N Engl J Med. 2017; 376(18):1713-1722.

31. Giugliano RP, Mach F, Zavitz K, et al. Cognitive function in a randomized trial of evolocumab. N Engl J Med. 2017;377(7): 633-643.

32. Toth PP, Descamps O, Genest J, et al. Pooled safety analysis of evolocumab in over 6000 patients from double-blind and open-label extension studies. Circulation. 2017;135(19):1819-1831.

33. Braamskamp MJ, Kusters DM, Avis HJ, et al. Long-term statin treatment in children with familial hypercholesterolemia: more insight into tolerability and adherence. Paediatr Drugs. 2015; 17(2):159-166.

34. van der Graaf A, Cuffie-Jackson C, Vissers MN, et al. Efficacy and safety of coadministration of ezetimibe and simvastatin in adolescents with heterozygous familial hypercholesterolemia. J Am Coll Cardiol. 2008;52(17):1421-1429.

35. Talmud PJ, Shah S, Whittall R, et al. Use of low-density lipoprotein cholesterol gene score to distinguish patients with polygenic and monogenic familial hypercholesterolaemia: a case-control study. Lancet. 2013;381(9874):1293-1301.

36. Besseling J, Kindt I, Hof M, Kastelein JJ, Hutten BA, Hovingh GK. Severe heterozygous familial hypercholesterolemia and risk for cardiovascular disease: a study of a cohort of 14,000 mutation carriers. Atherosclerosis. 2014;233(1):219-223. 